\title{
Criminologie
}

\section{Les petits commerçants victimes de vol à main armée en quête de justice}

\section{Hélène Manseau et Hélène Grenier}

Volume 12, numéro 1, 1979

Justice et public

URI : https://id.erudit.org/iderudit/017099ar

DOI : https://doi.org/10.7202/017099ar

Aller au sommaire du numéro

Éditeur(s)

Les Presses de l'Université de Montréal

ISSN

0316-0041 (imprimé)

1492-1367 (numérique)

Découvrir la revue

Citer cet article

Manseau, H. \& Grenier, H. (1979). Les petits commerçants victimes de vol à main armée en quête de justice. Criminologie, 12(1), 57-65.

https://doi.org/10.7202/017099ar d'utilisation que vous pouvez consulter en ligne.

https://apropos.erudit.org/fr/usagers/politique-dutilisation/ 
Hélène Manseau Hélène Grenier

A l'automne 1977 paraissait un rapport de recherches portant sur des victimes de vol à main armée dans la région montréalaise ${ }^{1}$. L'étude fut effectuée dans un cadre de recherches sur les attitudes du public canadien envers la criminalité.

La recherche visait à connaître, chez les personnes concernées par le vol à main armée, l'impact de la victimisation, sur leurs attitudes et opinions envers le système de justice canadien. Nous avons voulu cerner également leur perception du crime et du criminel à travers la confrontation dont ils furent l'objet.

Tout au cours de cette enquête, nous avons privilégié une approche qualitative des sujets et c'est sous forme d'entrevue semi-structurée que nous avons obtenu leur témoignage. Nous ne pouvons pas faire des généralisations à partir de nos résultats, étant donné que notre échantillon n'est pas représentatif et que notre approche fut qualitative plutôt que quantitative. Cependant, les témoignages recueillis, les résultats obtenus, après compilation des entrevues, furent parfois si troublants qu'ils méritent, selon nous, qu'on leur accorde une attention particulière.

La population spécifique touchée par cette étude se compose de propriétaires ou employés de petits commerces particulièrement victimogènes (tels les épiciers, les dépanneurs, les pharmacies...) qui ont été victimes d'un ou de plusieurs vols au cours des deux dernières années. Nous avons pu rencontrer ainsi quarante personnes auprès desquelles nous avons effectué notre enquête.

Donc, ce qui importe ici, c'est de nous interroger sur la signification du vol à main armée dans la perspective, cette fois, de ses victimes. Qu'est ce qu'un vol à main armée ? Quel est le rôle joué par les victimes? Quelles en sont les conséquences?

1. Les victimes de vol à main armée en quête de justice ». Rapport inédit d'une recherche effectuée par Hélène Manseau et Hélène Grenier sous la direction de Micheline Baril. G.R.A.C., rapport no 5, 185 pages, Université de Montréal. 
Quels problèmes immédiats créent-ils ? Quel est son impact global sur qui le subit?

\section{I - LA SCIENCE DU CRIME}

Habituellement, c'est un voleur novice et mal préparé qui entre en scène. Dans la majorité des cas, les agresseurs ont de 16 à 25 ans. Le déroulement de l'action est relaté par nos interlocuteurs d'une manière fort décousue, vu l'extraordinaire rapidité avec laquelle les événements s'y succèdent.

Ce qui surprend, c'est l'infinie variété de péripéties. Chaque scénario se déroule selon son rythme propre et sa logique interne. Il est impossible ici, sans simplifier, d'élaborer des typologies du vol qualifié. Et pourquoi ? Parce que le hold-up d'un petit commerce est avant tout une aventure très personnalisée, un mode de relations des plus dynamiques. Victimes et agresseurs sont généralement de parfaits étrangers l'un pour l'autre. Pourtant, durant les quelques secondes ou minutes que durera l'attaque, ils seront unis dans un même instinct de survie personnelle, chacun anticipant les réactions de l'autre et agissant en conséquence. Comme au jeu d'échecs, un simple coup peut faire échouer une savante stratégie.

La rapidité de l'échange entre les deux acteurs ne permet pas une stabilisation des rôles dans des modèles plus stéréotypés. Les réactions demeurent à un niveau primaire, instinctif, variant à l'infini en fonction des individus. C'est ainsi que le déroulement des événements oscille souvent entre la tragédie et la comédie. Même si les commerçants prévoient des stratégies d'action, le moment du crime fait surgir des réactions impromptues. Une arme sous le nez, l'agressé peut facilement « perdre les pédales » et provoquer chez l'agresseur des gestes de violence qui aboutissent à des conséquences désastreuses. Les voleurs étant généralement décrits comme nerveux et à l'affût des moindres faux pas.

Nous venons de voir que le hold-up est imprévisible. Il se produit au moment où l'on s'y attend le moins. Son modus operandi est déroutant et les réactions des acteurs sont inattendues. Même si les commerçants victimes rencontrés n'en étaient pas à leurs premières armes, cet événement continue toujours d'être aussi troublant. Environ $50 \%$ de nos interlocuteurs avaient déjà subi une moyenne de cinq vols à main armée au moment de 
l'entrevue et quelques-uns en avaient même subi plusieurs douzaines.

\section{I - LE MYTHE DE LA VICTIME COUPABLE}

La notion populaire d'un vague relent de culpabilité chez les victimes a été étudiée par certains criminologues. Mendelsohn, considéré comme le fondateur de la «victimologie \$ a basé sa typologie des victimes sur leur degré de culpabilité. Quelques années plus tard, en 1948, Von Hentig avançait que la victime façonne et moule le criminel. Plusieurs travaux empiriques ont été exécutés dans le but de vérifier cette affirmation. Ce sont les crimes violents qui ont fait l'objet d'études empiriques sur la relation criminel-victime. Dans le cas du vol à main armée, les chercheurs dont, entre autres, Normandeau (1968), Conklin (1972) et Curtis (1974), s'accordent pour dire qu'une faible proportion de ces délits ont été commis à l'instigation directe ou indirecte de l'agressé ; les pourcentages variant entre 10 et $20 \%$. Selon l'expression de Normandeau (1968), la collaboration de la victime, dans la situation de vol qualifié se décrit en termes de «tentationopportunité \$. Le voleur exploiterait la négligence, l'insouciance ou la provocation indirecte de la victime.

Jusqu'à quel point nos interlocuteurs ont-ils servi d'appâts, inconscients ou volontaires, à leurs agresseurs ?

Tout d'abord, il nous apparait que le fait d'avoir des heures de fermeture tardive soit d'un attrait particulier pour les assaillants. La plupart des commerçants que nous avons rencontrés ont des heures d'ouverture prolongées. Il est intéressant de constater qu'à l'origine ces petits commerces auraient agi ainsi afin de sauvegarder leur existence. Souvenons-nous du fait que ces heures d'ouverture et ces types de commerce ont commencé à voir le jour avec l'avènement des super-marchés. Autrefois, à Montréal, le petit commerce pouvait survivre financièrement sans devoir assumer un service le soir. Pour concurrencer les gros marchands, les petits commerces ont affiché des heures d'ouverture spécifiques et accommodantes. D'ailleurs la plupart de ceux rencontrés ont emprunté un nom qui va dans ce sens : "dépanneur ».

Or, peut-on prétendre qu'un citoyen pour des buts de survie économique, dans des conditions tout à fait loyales puisse être celui que l'on tient tacitement responsable d'un acte criminel ? Nous sommes portés à croire que non. 
Nous sommes également enclins à penser que le fait de gérer une entreprise familiale, donc avec un personnel vulnérable et réduit, puisse constituer un élément provocateur. Il importe de distinguer, en fait, entre provocation et vulnérabilité, ce qui a souvent été négligé.

Nous avons vainement essayé de détecter chez nos victimes des traces de responsabilité à l'égard de leurs assaillants. Ce qui ressort plutôt, c'est un sentiment généralisé d'impuissance. À l'exception de certains qui, après plusieurs expériences traumatisantes, décidèrent d'assurer eux-mêmes leur protection, les commerçants utilisent plusieurs systèmes d'alarme, sans pour autant être à l'abri d'un vol dont ils sont victimes chaque année, ou presque.

Ceux qui, accablés par une suite importante de vols décident de se faire justice en bravant l'agresseur, le font à leurs risques et périls. Nous avons vu que cette réaction défensive peut provoquer la violence de l'agresseur. Mais, encore là, peut-il être question de véritable provocation? Les pertes subies et les risques encourus nous font plaider en faveur de l'autodéfense plutôt qu'en fonction d'une provocation de la part des victimes; même dans les cas où, pour se défendre, ils en venaient à sembler se transformer en agresseurs. Nous avons pu d'ailleurs constater que ceux-là qui contestent ouvertement l'agression en dégainant leur pistolet s'étaient vu attaquer physiquement lors des assauts. En somme, lorsqu'ils décident de manifester de la violence à l'égard de l'agresseur, c'est vers eux que la violence se retourne dans la plupart des cas. Ce phénomène, comme bien d'autres, nous amène à être sceptique à l'égard de l'hypothèse de la victime coupable en ce qui a trait aux petits commerçants tout au moins ceux que nous avons rencontrés.

\section{III - LES DÉGÂTS MATÉRIELS PROVOQUÉS PAR LE VOL Ȧ MAIN ARMÉE}

La première perte subie et la plus évidente est celle de la somme d'argent volée. Les montants dérobés variaient entre $\$ 25$ et $\$ 1800$. S'ajoutent à cela des dégâts matériels lorsque les visites des voleurs furent plus violentes (vitres à changer, vandalisme, etc.).

De plus, certains agresseurs ne se contentent pas de voler. On ligote la victime, on l'enferme, on lui passe un sac sur la tête. Dans d'autres cas, les coups furent plus sérieux : coups de crosse 
de fusil dans l'estomac, brûlures et balle tirée dans la main. Ces coups entraînant à leur tour des pertes directes et indirectes : incapacité temporaire de travailler, pertes financières de tous genres, hospitalisation. Le hold-up provoque parfois un choc psychologique violent. Quelques-uns de nos interlocuteurs ont d'ailleurs vendu leur commerce soit parce qu'ils refusaient de prendre le risque à nouveau de vivre une expérience aussi pénible, soit que leurs revenus ne leur permettaient plus d'assurer les coûts d'un vol éventuel.

Il y a aussi les pertes indirectes, entre autres les primes d'assurance, les coûts des systèmes d'alarme, caisse électronique, chien policier et autres moyens de protection.

En plus, une image des plus pessimistes du système de justice s'ajoute aux dégâts matériels des victimes, à titre de conséquence de l'action criminelle.

\section{IV - LA PERCEPTION DU SYSTÈME DE JUSTICE}

C'est en recevant le subpœna que le commerçant commence à être désenchanté. Généralement, il ne réalise pas que l'appel instinctif porté à la police après le crime sera interprété comme une plainte. À partir de cet instant, il perd définitivement le contrôle de la situation. C'est ainsi que $\mathbf{2 2}$ de nos répondants avaient été invités à comparaître en cour et c'est surtout là que les illusions des victimes, face à la justice, s'étiolent. Les gens sont impressionnés, nerveux mais se rendent compte du jeu qui se passe dans les coulisses. Car, pendant que les témoins font les cent pas, les procureurs, eux, sont actifs. Puis, les trois coups sont sonnés et c'est alors que le témoin-victime est appelé à témoigner. Ils sont gênés et parfois même décontenancés de se retrouver en compagnie de leur agresseur. Cette gêne se transforme parfois en crainte de représailles. Les commerçants ont peur et cette peur influence sûrement le degré de leur collaboration avec la cour. Fort souvent, en outre, la cause est remise, un témoin ne s'étant pas présenté, et de remise en remise, le commerçant se sent de plus en plus lésé. Il impute souvent aux avocats la responsabilité de ses frustrations, le juge étant perçu comme ayant un rôle effacé.

$\mathrm{Ce}$ qui revient le plus souvent dans les témoignages des victimes, c'est l'opinion que l'expérience de la cour est perçue 
comme très négative, inutile et dévalorisante. La plupart se sentent à certains moments attaqués : "Ils t'interrogent comme si c'était toi qui étais le véritable voleur. Tu n'es plus la victime... tu es l'accusé. \$

En somme, les commerçants estiment que, devant le tribunal, les criminels sont mieux traités qu'eux-mêmes. Accusé pour accusé, l'agresseur a au moins l'avantage de pouvoir recourir aux services d'un avocat. Tout le monde y trouve son profit sauf la victime.

Tout procès se termine par le prononcé de la sentence. Lorsque le rideau tombe, le commerçant-victime ne connaît souvent pas la sentence de son agresseur, le procès étant encore en instance de procédure. Mais dans la plupart des cas, le marchand ignore l'issue des audiences, n'étant jamais convoqué au prononcé de la sentence. Si quelques commerçants-victimes sont satisfaits du déroulement des procédures devant le tribunal, la grande majorité exprime la déception, des frustrations, de l'amertume, si ce n'est de l'agressivité. Les commentaires touchent trois thèmes principaux : les pertes de temps et d'argent, les machinations hors cour et le traitement reçu devant le tribunal. La lenteur du système des tribunaux exaspère les commerçants qui se voient obligés de se présenter en cour à plusieurs reprises perdant ainsi un temps précieux, la plupart travaillant de 12 à 14 heures par jour. Ils voient le procès comme un événement à grand déploiement orchestré d'avance.

La connaissance expérientielle semble étroitement liée à la nature, la richesse et la couleur des opinions sur les tribunaux. Ce qui nous a frappé c'est que celui qui a été appelé à témoigner non seulement exprime des idées plus précises mais a aussi une perception plus négative que les individus qui n'ont pas vécu d'expérience à la cour. Ces derniers ont peu d'opinion sur la cour, les avocats, les juges, les procédures. Au cours de l'entrevue, ils soulevaient rarement le sujet de façon spontanée. Leurs impressions sont floues, stéréotypées et souvent plus positives. En fait, ils n'ont jamais vraiment réfléchi à la question et ne sont pas spécialement intéressés à en parler.

Qu'ils critiquent les juges, les avocats, le jury, oui, c'est un fait, mais ils sont encore plus véhéments à l'égard du système. Évidemment, c'est plus commode de blâmer le système, mais 
aussi, l'adulte réaliste et peu averti, au fait, des technicalités judiciaires, ne se sent pas compétent pour distribuer les reproches. Il exprime une insatisfaction généralisée imputable tantôt aux agents, tantôt à l'organisation elle-même gigantesque machine anonyme.

Pour plusieurs, le concept de justice en est un très abstrait, ne faisant relation à rien de tangible. C'est un terrain vaste, et relevant du domaine des spéculations philosophiques. D'autres nous parlent des deux justices ; celle des riches et celle des pauvres : - La justice n'est pas égale pour tout le monde * (M. Tylipe); * De la justice, y en a pour quelques-uns, y en a pas pour d'autres, (Mme Zinnia).

Et pourtant, les opinions sont souvent contradictoires. On déplore l'existence d'une * justice * injuste et inefficace, mais on prône aussi sa survie et sa nécessité. De fait, une lacune majeure existe au niveau de l'articulation du témoignage; très peu de répondants parlaient d'amélioration possible, rares sont ceux qui formulaient des propositions pour faire changer ce système qu'ils critiquent.

\section{$V$-LIMPACT DE LA VICTIMISATION SUR LES ATTITUDES ET OPINIONS DES PETITS COMMERÇANTS}

En présence de conjectures pour le moins insécurisantes, les quarante commerçants qui ont participé à notre recherche apparaissent fort préoccupés par la nécessité de réintroduire dans notre système pénal le processus de la punition, comme élément dissuasif de la criminalité.

Devant l'angoisse que peut susciter la présence répétée d'agresseurs dans leur établissement, ils en viennent à une sorte de retour à un ancien système plus sévère et régi selon des règles plus strictes. C'est en quelque sorte un besoin de prévision : une volonté de retrouver des normes assez définies pour que les membres de la société puissent comprendre et se situer en dehors de toute confusion. Un des facteurs corroborant clairement ce point de vue pourrait certes être la répugnance qu'ils manifestent à l'égard des libérations conditionnelles. C'est comme si le chaos occasionné par la Commission contre eux-mêmes d'un acte criminel nécessitait des précisions importantes quant au règlement du conflit suscité. On aurait besoin de connaître les modalités précises de règlementation du conflit. 
Néanmoins l'on ne peut conclure des propos des victimes que nous avons rencontrées qu'elles soient pour ainsi dire punitives au sens littéral du terme. Nous avons vu que leurs exigences quant aux punitions relatives à leurs agresseurs, loin d'être répressives, se veulent constructives avant tout. Nous entendons par là que leur confiance en l'ergothérapie et leurs espoirs quant à la réadaptation et la réhabilitation ne démontrent pas des attitudes essentiellement punitives. Malgré tout, leurs discours laissent indubitablement une place prépondérante à la punition mais cette punition n'est pas définie ou perçue comme une forme de vengeance ou comme une façon de punir pour punir.

Tout laisse croire, au contraire, à un besoin de récupération de la criminalité par la recherche de traitements adéquats et visant à contrer à tout prix la recrudescence de la criminalité. Il est étonnant de constater la tolérance que les victimes témoignent à l'égard des jeunes et des criminels « accidentels ». C'est dans cet esprit qu'il est important de ne pas privilégier l'idée de châtiment dans leur volonté de punir. À la lumière de ce développement, on peut aussi mieux comprendre le fait qu'elles soient tentées d'accuser le système actuel plutôt que de se révolter contre le crime et le criminel. Si l'on conçoit que la criminalité puisse provenir d'un manque de sévérité du système, on est porté avant tout à critiquer ce système lorsqu'il est question d'une recrudescence alarmante du phénomène criminel.

Par ailleurs, si on veut bien regarder les principales attitudes de nos interlocuteurs, il est fondamental de prendre note de l'intérêt qu'ils portent au travail. La notion de travail est à la base d'un grand nombre de leurs raisonnements. Ainsi, ils vont jusqu'à tolérer une forme de malhonnêteté, chez les avocats par exemple, en évoquant le fait que c'est une exigence inhérente à leur travail. Cette notion est d'ailleurs omniprésente quant à leur façon de concevoir la réhabilitation et leur quasi-obsession peut sûrement être en partie explicable, si l'on considère qu'eux-mêmes sont des travailleurs qui dépassent les normes horaires habituelles, car ils travaillent au-delà de quatre-vingts heures par semaine. Cette situation n'est sans doute pas de nature à insensibiliser le commerçant au fait d'être dépossédé de ses biens, loin de là ! $\mathrm{Au}$ contraire nos marchands, dans la plupart des cas, sont extrêmement indignés par le fait même du vol de leurs biens. D'ailleurs, dans leurs propos, la notion de propriété revient fréquemment. 
C'est peut-être à cause de cela que l'argent occupe une place prépondérante dans leur façon de concevoir la justice.

Il a été intéressant de constater à un moment donné de notre recherche que les interviewers étaient unanimes à reconnaître le fait de restituer les biens volés aux victimes rencontrées puisse être un facteur qui engendrerait un impact fort marquant sur l'opinion que se font ces derniers de notre système de justice criminelle. Mais cette idée ne peut évidemment être soulevée qu'à titre d'hypothèse. Ce qu'il importe pour nous de savoir, ici, c'est que les pertes financières encourues sont apparues au cours de nos entretiens comme un facteur prégnant et non négligeable. Dans cet esprit, il faut aussi mentionner que l'individualisme nous semble être une caractéristique particulière des gens interrogés. Pas un seul d'entre eux n'a émis l'idée de projets communs de protection, par exemple. La société qu'ils recherchent accorderait de la valeur et des droits aux individus avant tout; une sorte de solidarité sociale est presque inexistante dans les volontés qu'ils nous ont décrites. Le respect des droits individuels est donc un aspect essentiel de leur pensée.

\section{CONCLUSION}

A ce stade-ci, il s'avère très difficile de distinguer parfaitement l'origine des représentations qu'ont les petits commerçants concernant la qualité du système de justice criminelle. Il est à toute fin pratique impossible de disséquer l'influence de la victimisation sur leurs opinions. Cependant nous pouvons dire que leur vision à eux du système de justice est pour le moins pessimiste et que la dose de pessimisme augmente proportionnellement au nombre de crimes qu'ils subissent et, malheureusement aussi, en fonction du nombre d'étapes qu'ils traversent à l'intérieur du système de justice criminelle.

Pour nous, chercheurs, c'est l'immense impuissance dont ils ont témoigné qui nous a le plus impressionnés. Impuissance liée à leur condition de victime d'acte criminel d'abord puis, de surcroît, impuissance devant l'oppression qu'ils ont vécue à l'intérieur du système. 